\title{
Non-surgical treatment for early prostate cancer
}

\author{
${ }^{1} \mathrm{AB}$ Law, ${ }^{2} \mathrm{DB}$ McLaren \\ ${ }^{1}$ Specialist Registrar Clinical Oncology; ${ }^{2}$ Consultant Clinical Oncologist, Department of Oncology, Western General Hospital, Edinburgh, UK
}

\begin{abstract}
Prostate cancer incidence is rising due to the ageing population and increased public and doctor awareness. The role of screening is still not clear due to the large number of asymptomatic men who would need to be screened and treated to prevent one death. Discussion of all treatment options should be undertaken, with the patient having the opportunity to meet a clinical oncologist and urological surgeon. Treatment options include active surveillance, external beam radiotherapy, brachytherapy and surgery. Low-dose rate brachytherapy involves the permanent insertion of radioactive seeds (half-life 60 days) under ultrasound guidance. It is a good option for many men as impotence and incontinence rates are lower than for surgery and it has reduced hospital costs and time off work and high rates of relapse-free survival $(90-95 \%$ in low-risk disease). External beam radiotherapy offers a good treatment for men with more locally advanced disease and men who do not want to undergo an anaesthetic. New developments allow higher doses of radiotherapy to be given with reduced relapse rates and reduced toxicity to neighbouring structures such as bowel and bladder. High-dose rate brachytherapy involves the temporary insertion of applicators into the prostate so that a high energy source can temporarily be fed into different positions in the prostate, ensuring a high dose to the prostate gland but minimising dose to the bladder and bowel. It can be used as monotherapy or in combination with external beam radiotherapy.
\end{abstract}

Correspondence to AB Law, Department of Oncology, Western General Hospital, Crewe Road South, Edinburgh EH4 2XU, UK

tel. +44 (0) I3I 5372215

e-mail alastair.law@luht.scot.nhs.uk

KEYWORDS Brachytherapy, external beam radiotherapy, prostate cancer

DECLARATION OF INTERESTS No conflict of interests declared.

\section{INTRODUCTION}

Prostate cancer is the most common cancer in men, but for localised disease there is continued controversy about whether treatment should be immediate or deferred, which type or combination of treatments is optimal and what the role of screening is.

The diagnosis of prostate cancer is normally made through transrectal biopsies (usually at least five cores from both sides of the prostate gland) under ultrasound guidance. The pathologist will report the number of cores involved and the pathological grading (using the Gleason score, adding the two most common grades of tumour detected), which normally ranges from 6 (most differentiated) to 10 (poorly differentiated). After the diagnosis of prostate cancer (whether through presentation with symptoms or screening), a decision needs to be made as to which patients require treatment. Staging with a bone scan and magnetic resonance imaging or computed tomography of the pelvis should be done in all cases considered for radical treatment, except those with a low risk of spread outside the prostate, such as men with prostate-specific antigen (PSA) $<10 \mathrm{ng} / \mathrm{ml}$ and Gleason score $\leq 6$.

The patient's general health needs to be considered and most centres only consider radical treatment of localised disease if life expectancy is greater than ten years as most men would not become symptomatic during this period. For those with apparently localised disease, the Gleason score, local tumour extent (T stage), presenting PSA level, age and general health will all be taken into account.

Prostate-specific antigen screening is also a controversial area and a recent study has shown that PSA screening reduces the ratio for death from prostate cancer in the screening group to 0.80 (95\% confidence interval, $0.65-$ 0.98 ; adjusted $\mathrm{p}=0.04) .{ }^{\prime}$ The absolute risk difference was $0.7 \mathrm{I}$ deaths per I,000 men. Thus, I,4I 0 men would need to be screened and 48 additional cases of prostate cancer would need to be treated in order to prevent just one death from prostate cancer. At present, few countries have adopted screening.

Having decided that local treatment is indicated, there is the question of which treatment is best for the individual patient. There is a choice between radiotherapy, either external beam radiotherapy alone, in combination with brachytherapy or prostate brachytherapy alone, or radical surgery. As with many cancers where both surgery and radiotherapy are options for treatment, there are no randomised trials comparing outcomes, but most series of the different treatments produce very similar results. For low-risk cancers (those with a Gleason score $\leq 6$ and PSA $<10 \mathrm{ng} / \mathrm{ml}$ ) active surveillance is an option in all age groups, with regular PSA and repeat biopsies and consideration of treatment if PSA or tumour grade changes.

Traditionally surgery has been used in younger men with smaller tumours and less aggressive pathology and lower 
PSA results. The main long-term side effects from surgery are urinary incontinence and impotence, although the rates from laparoscopic nerve-sparing operations are lower.

\section{NON-SURGICAL TREATMENTS}

\section{Interstitial (low dose rate) brachytherapy}

Prostate brachytherapy is the fastest-growing radical treatment for early prostate cancer in Europe and the USA. The patient is anaesthetised and a radioactive source, usually composed of iodine- 125 , is inserted through the perineum into the prostate using ultrasound guidance. The seeds are left in the prostate gland permanently and emit radioactivity with a half-life of approximately 60 days. In our series from Edinburgh, which serves as the referral centre for the east coast of Scotland, there were five-year PSA relapse-free rates of $94 \%$ for low-risk prostate cancer and $81 \%$ for intermediate-risk cancers. ${ }^{2}$

The advantage of this procedure is that the anaesthetic is short and there is a lower acute risk of complications, such as infection and bleeding, compared with surgery. It is best suited to men who do not have grossly enlarged prostate glands (below $50 \mathrm{~cm}^{3}$ ) and who do not have a lot of urinary symptoms. The procedure is done either as an outpatient or requires a one-night hospital stay. Most men experience cystitis, peaking at three months, but the urinary incontinence rate is less than $1 \%$ and $65-70 \%$ of men under the age of 70 who were previously potent remain potent after the procedure. The majority are back at work and have resumed normal activity within a week of the procedure, which compares favourably with open and laparoscopic surgery.

Another benefit of brachytherapy is cost, with the best data on relative costs from the US. There, health insurance providers calculate that prostate brachytherapy is $25 \%$ cheaper than radiotherapy and $50 \%$ cheaper than prostatectomy. The costs calculated were only direct costs and do not take into account costs incurred to the patient due to prolonged periods away from work after surgery or daily travelling for radiotherapy.

\section{High-dose rate (HDR) brachytherapy}

In HDR brachytherapy, applicators (tubes) are placed in the prostate under general or spinal anaesthetic through the perineum. The applicators are then connected to a machine and a radioactive source, usually iridium, is fed in and out of the different applicators to achieve a high and homogeneous dose to the prostate gland while minimising the dose to neighbouring structures such as the bladder and rectum. The advantage of this technique over external beam radiotherapy is that a much higher dose can be achieved with a greater tumoricidal effect. The use of HDR brachytherapy with external beam radiotherapy lowers the number of fractions of radiotherapy, thereby reducing costs and patient visits.
High-dose rate brachytherapy can be used as monotherapy for low-risk cancers or in combination with external beam radiotherapy for intermediate- or high-risk cancers. A randomised trial has shown improved rates of recurrence (as measured by a rise in PSA) when the combination of HDR brachytherapy and external beam radiotherapy was used over external beam radiotherapy alone. ${ }^{3}$

\section{External beam radiotherapy}

In this treatment the patient attends as an outpatient on weekdays over a number of weeks (usually between four and eight weeks). The advantage of this technique is that the patient does not need any anaesthetic and it can also be used in more advanced stages such as when there is extracapsular spread of the tumour outside the prostate. In cases of extracapsular spread, surgery would be likely to leave involved margins and it is technically difficult to position radioactive sources in brachytherapy to get optimal coverage of disease outside the capsule.

Recent innovations in external beam radiotherapy are the use of more precise targeting of the prostate, using 'fiducial' seeds that are inserted into the prostate prior to starting. These marker seeds are then visualised at the time of radiation delivery to ensure the prostate is targeted optimally as the position of the prostate can change with rectum and bladder filling. Toxicity is low, with long-term bowel and bladder side effects usually I- $3 \%$.

In recent years intensity-modulated radiotherapy has become increasingly used in UK centres. This technique allows higher doses to be delivered to the prostate gland while minimising dose to the rectum and bladder. Recent studies have shown that higher doses of radiotherapy, for example 78 Gray (Gy) compared with $70 \mathrm{~Gy}$, have improved relapse-free survival rates.

Hormone treatment is commonly used in combination with radiotherapy prior to ('neoadjuvant') starting radiotherapy. This has been shown to reduce the relapse rates and the effect is thought to be related to shrinking the prostate gland and radiosensitisation of the cells. There is also an additional $5 \%$ survival advantage from giving additional hormone treatment for 2-3 years after radiotherapy, particularly in more advanced cases.

\section{CONCLUSION}

Prostate cancer incidence is rising due to the ageing population and increased public and doctor awareness. Discussion of all treatment options should be undertaken, with the patient having the opportunity to meet a clinical oncologist and urological surgeon. Improved results and reduction in side effects have been obtained with the development of new techniques such as brachytherapy and more advanced external beam radiotherapy techniques. 


\section{REFERENCES}

I Schröder FH, Hugosson J, Roobol MJ et al. Screening and prostatecancer mortality in a randomized European study. $N$ Engl J Med 2009; 360: | 320-8. doi: I0.1056/NEJMoa08I0084

2 Law AB, Kerr G, Howard G et al. Prostate cancer treated with permanent seed implantation - the East Scotland experience. Poster presentation. UK and Ireland Brachytherapy Conference, March 2010. Available from: http://www.prostatebrachytherapy. org.uk/abstract submissions.htm

3 Hoskin PJ, Motohashi K, Bownes $\mathrm{P}$ et al. High dose rate brachytherapy in combination with external beam radiotherapy in the radical treatment of prostate cancer: initial results of a randomised phase three trial. Radiother Oncol 2007; 84:II4-20. doi:10.1016/j.radonc.2007.04.01 I

\section{FURTHER READING}

- Pollack A, Zagars GK, Starkschall G et al. Prostate cancer radiation dose response: results of the M.D. Anderson phase III randomized trial. Int J Radiat Oncol Biol Phys 2002; 53:1097-105. doi: 10.1016/ S0360-3016(02)02829-8

- Schröder FH, Roach M 3rd, Scardino P. Management of prostate cancer. N Engl J Med 2008; 359:2605-9. doi:10.1056/ NEJMclde080549I

- Widmark A, Klepp O, Solberg A et al. Endocrine treatment, with or without radiotherapy, in locally advanced prostate cancer (SPCG-7/SFUO-3): an open randomised phase III trial. Lancet 2009; 373:30I-8. doi:I0.10I6/S0 I40-6736(08)6I8I5-2. Erratum in: Lancet 2009; 373): II 174.

\section{SELF-ASSESSMENT QUESTIONS}

I. In the treatment of a potent patient with prostate cancer, incontinence rates are likely to be highest after which one of the following treatments?

A. Open prostatectomy.

B. Laporoscopic nerve-sparing surgery.

C. Low-dose rate brachytherapy.

D. Combination of high-dose rate brachytherapy and external beam radiotherapy.

E. External beam radiotherapy alone.

2. Which one of the following is the approximate number of men that would need prostate-specific antigen (PSA) screening in order to prevent one death from prostate cancer?

A. 10.

B. 40 .

C. 400 .

D. 1,400 .

E. 4,000 .

3. Which one of the following would be the best treatment for a fit 65 -year-old patient with a localised prostate cancer (pathological scoring, Gleason 7) with gross extracapsular spread (stage T3a) but no distal spread?

A. Surgery.

B. Brachytherapy using iodine seeds.

C. A combination of hormones and external beam radiotherapy.
D. Hormones alone.

E. Active surveillance with PSA monitoring and consideration of treatment if PSA rose.

4. Which one of the following is the chance of being disease-free (measured by prostate-specific antigen level) approximately 5 years after radical treatment for a low-risk prostate cancer?

A. $90-95 \%$.

B. $80-85 \%$.

C. $70-75 \%$.

D. $60-65 \%$.

E. $50-55 \%$.

5. Which one of the following is the incidence of late significant gastro-intestinal toxicity such as rectal bleeding or fistula formation after brachytherapy or external beam radiotherapy for prostate cancer?

A. $1-3 \%$.

B. $10 \%$.

C. $20 \%$.

D. $30 \%$.

E. $50 \%$.

This paper was originally published as part of the Oncology module in the RCPE Online Continuing Medical Education Programme. Online CME, including the anwers to these questions, is available to Fellows and Members at: http://www.rcpe.ac.uk 